Obstet Gynecol Clin North Am. 2006 March ; 33(1): 1-11. doi:10.1016/j.ogc.2005.12.004.

\title{
Epidemiology of Myomas
}

\author{
Mark Payson, MD ${ }^{\mathrm{a}, \mathrm{b}}$, Phyllis Leppert, MD, PhD ${ }^{\mathrm{a}, \mathrm{b}}$, and James Segars, MDa,b, \\ James Segars: segarsj@mail.nih.gov \\ aReproductive Biology and Medicine Branch, NICHD, National Institutes of Health, Building 10, \\ CRC, 1E-3140, 9000 Rockville Pike, Bethesda, MD 20892, USA \\ bDepartment of Obstetrics and Gynecology, Uniformed Services University of the Health \\ Sciences, 8900 Wisconsin Avenue, Bethesda, MD 20814, USA
}

\begin{abstract}
Uterine leiomyomas (or fibroids) are a prevalent and morbid disease. Leiomyomas place an enormous health care burden on American women, and disproportionately affect African American women. Despite their prevalence, the disease has remained enigmatic, with the incidence, natural history, and progression incompletely understood [1]. A scholarly examination of the epidemiology of fibroid disease faces five sizeable challenges.
\end{abstract}

First, is fibroid disease a single entity or more than one disease? It is now appreciated that leiomyoma development is a phenotype featured in several genetic diseases; leiomyoma encountered clinically may not represent a single disease entity. Most obviously, disease progression and outcome might vary between the different types of disease, perhaps in different ethnic groups. The appreciation that there may be different phenotypes of fibroid disease is suggested by recent molecular profiling studies, described later in this article.

Second, there is not a widely accepted, standardized classification system for leiomyomas; fibroids are different sizes and occur in different areas of the uterus. The absence of a scoring system to classify disease makes comparative assessment of disease problematic. The inability accurately to classify disease stage compromises studies of disease epidemiology.

Third, the incidence of disease (fibroids) varies as women age and with race. If these variables are not taken into consideration or carefully controlled, it is easy to draw false conclusions, or to be misled by the confounding variables that are age- or race-dependent and not an element of the fibroid evolution per se.

Fourth, diagnostic methods used to detect disease vary in sensitivity and specificity, and some are notably operator-dependent. This fact further obfuscates assessment of disease progression and comparison across studies.

*Corresponding author. Reproductive Biology and Medicine Branch, NICHD, National Institutes of Health, Building 10, CRC, 1E-3140, 9000 Rockville Pike, Bethesda, MD 20892.

The opinions or assertions are the private views of the authors and are not to be construed as official or as reflecting the views of the Department of Health and Human Services. 
Fifth, the incidence of fibroids that are sonographically detectable, but asymptomatic, is remarkably high, and in fact encompasses most American women by the age of menopause. Studies that simply use self-reporting underestimate prevalence of disease in a considerable number of patients with leiomyomas, and tend to draw incorrect conclusions. This form of bias may cause the detrimental nature of fibroids to be overemphasized or symptoms to be assigned incorrectly to leiomyomas. Stated differently, the medical literature is naturally biased toward assignment of clinical conditions to fibroid disease, a problem compounded by the fact that few studies have included appropriate age-matched control groups.

Bearing in mind these formidable obstacles, this article reviews the epidemiology of uterine fibroids. Clues to the etiology of fibroids may be gleaned by identification of individuals at risk and elucidation of risk factors. Furthermore, identification of modifiable risk factors may lead to strategies for prevention.

\section{Prevalence in different populations}

An oft cited study from the United States assessed the prevalence of fibroids in a population of patients undergoing tubal sterilization [2]. The prevalence in white women was 9\%, and in African American women 16\%. Interestingly, only one third of the women who had fibroids diagnosed during their tubal procedure had previously been given a diagnosis of fibroids, indicating that fibroids had either not been detected on previous examinations or that the patients had not reported sufficient symptoms to have been diagnosed. This fact emphasizes that the prevalence of fibroid symptoms reflects a fraction of the overall prevalence of disease.

The best designed studies examining overall prevalence have applied ultrasound diagnosis to a randomly sampled population. In another study from the United States [3] 1364 women 35 to 49 years old were screened by ultrasound. A third of the women had already been given a diagnosis of fibroids, and half of those who had not had a previous diagnosis had ultrasound evidence of fibroids. The cumulative incidence of fibroids by age 50 included most American women, almost $70 \%$ for whites and $80 \%$ for African Americans. Included in this number is $19 \%$ of women who did not have a "focal" fibroid identified, but simply had diffusely heterogeneous echo patterns indicative of fibroids. It is possible that not all of these patients had fibroids, that some had adenomyosis or simply myometrial contractions; however, even if this subset is excluded, uterine fibroids were still found in most cases. By the age of menopause in America, the presence of uterine fibroids seems to be the norm, not the exception.

The striking increased prevalence of disease in African American women brings into question the incidence of fibroids in populations in Africa, and whether the high prevalence of disease might reflect a genetic predisposition, or conversely diet or other environmental influences. Few studies have specifically addressed this point. In a Nigerian study the number of hospital admissions that could be attributed to fibroids was examined. Although not controlled for the population, $13.4 \%$ of new gynecologic admissions in Nigeria were admitted as a direct result of fibroids [4]. 
Fibroid disease seems to be less prevalent in European populations. In a German study began in 1998 [5], the German Cohort Study, a questionnaire-based survey of women's health among 10,241 women, the incidence of fibroid disease was only 12.7 per 100,000 years. If prevalence is calculated from their numbers, it seems to be surprisingly low at $5 \%$. This does not include, however, an equal number of patients who responded that they were given a diagnosis of "benign tumors of the uterus," which presumably were also fibroids. Including these patients, the prevalence doubles to $10.7 \%$. This reflects the number of women of mean age 39.6 who reported having been given those diagnoses, which certainly underestimates asymptomatic or undiagnosed fibroid burden. Despite these methodologic limitations, the results suggest that fibroid disease may be less common in central Europe.

The Seveso Women's Health Study [6] followed a cohort of women in Italy. In this study of 341 women aged 30 to 60 with a uterus, the incidence of ultrasonographically detectable fibroids was $21.4 \%$. This provides one of the best prevalence estimates for fibroids in a European population because the presence or absence of fibroids was determined independent of symptomatology.

A Swedish study also using ultrasound for detection of disease reported a relatively low prevalence of fibroids [7]. Five hundred fifty-four women aged 25 to 50, all Swedish citizens, were randomly selected from the national population register and asked to join the study; three quarters accepted. Fibroids were diagnosed in $3.3 \%$ of 25 to 32 year olds and in $7.8 \%$ of the 33 to 40 year olds.

Although not specifically addressing the population prevalence of disease, a Japanese study [8] examined the prevalence of fibroid disease in first-degree relatives of women undergoing surgery for fibroids. Thirty-one percent of women undergoing fibroid surgery reported a first degree relative with fibroids, as opposed to $15 \%$ of controls. Despite the limitations of a questionnaire-based survey about relatives' health status, it does provide information about the prevalence of fibroid disease in the Japanese population, and hints at a familial link.

\section{Prevalence in different race and ethnic groups}

Studies that have examined prevalence in different racial groups principally have been conducted in the United States. Because of the homogeneous nature of some of the populations in the samples referenced in the preceding section, it can be inferred that there may be significant differences in disease prevalence between women of different racial and ethnic background. Unfortunately, many reports do not specifically assess the incidence of fibroids between races within their population. In a study comparing prevalence of fibroids as diagnosed by ultrasound or hysterectomy across races only African Americans had an increased risk. Both Hispanics and Asians in the United States had risks similar to whites [9].

Several studies have shown an increased prevalence of fibroids in African Americans $[2,3,10,11]$. This disproportionate disease burden is manifest in the number of hysterectomies performed on African American women, $75 \%$ of which were performed for the indication of fibroids [12]. Studies in the United States revealed an incidence twofold to threefold greater in African American than white women. The likelihood of being diagnosed 
with fibroids was approximately 3\% per year for reproductive-age African American women [10]. In one ultrasound study that confirmed the high prevalence of fibroids in American women [3], there was an increased prevalence in African Americans. Fibroids were diagnosed at a younger age, were more often multiple, and tended to be larger in African Americans, with the cumulative incidence in excess of $80 \%$ by age 50 .

A carefully conducted case-control study [11] found self-reported African American heritage to be associated with a relative risk (RR) of fibroids of 9.4 compared with white women. The subjects in this study were women being seen for symptoms of fibroid disease and had fibroids confirmed either sonographically or surgically.

\section{Are there different types of fibroid disease?}

The literature of fibroid epidemiology treats fibroids as a single disease. Clinically, however, leiomyoma seem to exhibit at least three somewhat distinct phenotypes that although they have not been clearly defined, seem to carry different prognoses. Specifically, leiomyoma (1) may be single; (2) may be multiple and the uterus virtually peppered with multiple leiomyoma of varying size; and (3) may be found in association with adenomyosis, or alone. For example, in an interesting study [3] the diagnosis of fibroids was subcategorized as to whether fibroids were multiple or not. Seventy-three percent of black women had multiple fibroid tumors versus $45 \%$ of white women. Analysis at myomectomy suggests that fibroids from African Americans are larger than those from whites [1]. At myomectomy the affected uterus may feature a single tumor, or many tumors that are practically impossible to extirpate.

In some women the tumors are singular, and if removed rarely recur. In contrast in other women a uterus normalized by removal of several tumors may rapidly develop several more tumors within a few months. It is certainly possible that the myometrium of some women is more prone to develop fibroids and once fibroids develop they may grow more rapidly. Few studies [13] have reported the number of fibroids rather than simply their presence or absence. These markedly different clinical phenotypes of one pathologic condition beg the question: is there more than one type of fibroid disease?

The answer to this question seems to be, yes. Some leiomyoma clearly reflect underlying genetic predisposition to tumor development. For example, several reported genetic syndromes feature leiomyoma development, such as hereditary leiomyomatosis and renal cell cancer, Reed's syndrome, and Alport's syndrome [14-16]. The leiomyoma in such conditions are associated with known mutations, or abnormalities, such as fumarate hydratase in hereditary leiomyomatosis and renal cell cancer [17]. Leiomyoma associated with these rare genetic syndromes are grossly indistinguishable from common leiomyomata, but clearly these leiomyoma do not portend the same risk and disease, because in such a condition as hereditary leiomyomatosis and renal cell cancer, leiomyosarcoma may be a concern. Interestingly, preliminary reports of gene profiling studies suggest that fibroids from at least one syndrome, hereditary leiomyomatosis and renal cell cancer, may not resemble those of common leiomyoma (Mayers $\mathrm{C}$ and coworkers, unpublished data). 
Recent molecular profiling studies of fibroids lend support to the notion that fibroids may exist as different clinical phenotypes. That these two presentations may represent different types of fibroid disease is supported by gene profiling studies comparing fibroids from African Americans with whites [18]. This observation, coupled with the genetic syndromes mentioned, suggests that fibroids may represent a common smooth muscle response to several different disorders rather than a discrete disease. If this is indeed the case, it is important to elucidate the prevalence of different types of fibroids and the clinical course of the subtypes.

\section{Incidence at different ages and progression}

The incidence of pathologically diagnosed fibroids increases steadily with age [19]. At 25 to 30 years the incidence of fibroids is only 0.31 per 1000 women years, but by ages 45 to 50 the incidence has increased 20 -fold to 6.20 per 1000 women years. Advancing age increases the risk for fibroids many fold, and mirrors the understanding of the biologic development of fibroids: most grow in time and are expected to be diagnosed in greater numbers in older cohorts. A small sample of patients who had their fibroids followed-up ultrasonographically [20] saw an average growth of $1.2 \mathrm{~cm}$ in 2.5 years. The chance of being diagnosed with fibroids increases with age until about 50 years and then declines sharply $[8,11]$.

How quickly fibroids grow or recur was examined in a study of 145 women followed after abdominal myomectomy [21]. The recurrence of fibroids was diagnosed by ultrasound demonstrating fibroids at least $2 \mathrm{~cm}$ in diameter. The 5-year risk of recurrence was $62 \%$, with a $9 \%$ risk of an additional major surgery, an important number to be aware of when counseling patients for myomectomy. The recurrence risk was lower in patients with a solitary fibroid, a smaller total uterine size, and those who subsequently had a successful pregnancy.

Preliminary results from the ongoing NIEHS study [22] suggested that any fibroid documented on MRI does grow over time but at variable rates. Notably, no fibroids were seen that regressed during their observations [22]. The disappearance or shrinking seen in other studies [20] may be caused by the imprecise nature of ultrasound examinations, where a fibroid could be incorrectly mapped or other features of the myometrium, such as a myometrial contraction, may be incorrectly scored as a fibroid, which might then later be seen to disappear.

\section{Hormones and fibroids}

A case control study of 535 women who developed fibroids from a cohort selected from a family planning clinic found risk factors associated with events that could change estrogen levels [19]. In this study the diagnosis of fibroids was confined to those patients who had surgery to remove them. Oral contraceptive use decreased association with fibroids, with the RR decreasing in a dose-dependent fashion to the duration of oral contraceptive use. In the group with the longest use (>145 months), the risk of fibroids was half that of controls. What can be seen as a dramatic protective effect in these patients, however, can be attributed to the population studied. The patients with fibroids had a more difficult time achieving pregnancy, and would have less time during which they were using contraception. If the 
analysis had included any type of contraception, rather than just hormonal contraception, it might have shown a similar effect. Other studies [11] also reported a protective effect of oral contraceptives with a RR of 0.2 , but the confounders mentioned need to be kept in mind.

Of note, a large study that examined similar factors in 95,000 premenopausal nurses [23] found the only change in risk associated with oral contraceptives was age at first use; women who had first used oral contraceptives between 13 and 16 years of age had a significantly increased risk of uterine fibroids (RR 1.9). This could be attributed to either the known increased incidence of sexually transmitted disease among early initiators of sexual activity, or as a marker for metrorrhagia, which in and of itself could be a uterine irritant. Starting oral contraceptives at a young age could be a marker for other risk factors for fibroids, rather than a cause itself.

Obesity increased risk roughly $18 \%$ for each $10 \mathrm{~kg}$ increase, whereas two packs of cigarettes a day decreased the risk the same amount. Another study [11] showed an increased risk of 2.3 for fibroids for women in the upper quartile of body mass index. Although higher estrogen levels are present in obesity, one study that examined this variable [24] reported a reduced RR of fibroids in these patients (RR 0.6). This may be attributable to the difficulty of diagnosing fibroids in the obese population rather than a true protective effect, but it does not seem that obesity causes a marked increase in fibroid risk.

\section{Pregnancy and fibroids}

Several studies have shown a protective effect of pregnancy on the development of fibroids [2], with parity decreasing the risk of fibroids up to fivefold. These numbers may be deceptively high, however, given the known decrement in fertility attributable to fibroids. If a woman does not have fibroids she is more likely to have been pregnant and delivered a child, and because many of the studies look at parity rather than simply a history of being pregnant, the effect may be inflated even more, because fibroids not only interfere with implantation, but with successful delivery. There is biologic plausibility to the protective effect of progestins on fibroid growth, however, because in the Eker rat the incidence of fibroid disease was reduced with progestins [25].

The confounding effects that subfertility caused by fibroids has on analysis of effect of pregnancy on fibroids is well illustrated in a study examining the risk of fibroids and age at delivery [19]. A diagnosis of fibroids did not change the age of delivery of first children, but did change the age of last term delivery. Having a child later in life was conveyed as being protective, when in fact it may merely illustrate the fact that there was a greater disease burden of fibroids later in life and these women did not have their fertility affected at age19, but fertility was affected at age 38 .

\section{Menstrual cycle characteristics}

Fibroids are generally associated with an increased risk of heavy menstrual flow or a longer duration of menses [2]. The biologic plausibility was attributed to submucosal fibroids interrupting the normal endometrial development, or the burden of fibroids influencing normal myometrial contractility. Not all studies, however, have shown this relationship. In a 
cohort of women being followed independent of fibroid risk [6], 73 of who had ultrasonographically detectable fibroids, there was no significant difference in their menstrual cycle characteristics compared with controls. Because by definition these women were not selected for symptoms of fibroids, however, the sample size was not large enough to demonstrate the effect. This illustrates the point that disruption of the menstrual cycle is far from an inevitable outcome in women with fibroids. There is a bias to attribute symptoms to the tumors in women with fibroids.

\section{Hypertension}

A recent study demonstrated an intriguing link between diastolic blood pressure and fibroids [26]. In line with theories that show a graded response of diastolic blood pressures to atherogenesis, it was suggested that elevated blood pressure could cause injury or cytokine release in the uterine smooth muscle that promotes fibroid growth. In a 10-year analysis of more than 100,000 nurses there were 7466 diagnoses of fibroids by ultrasound or hysterectomy. After adjusting for age, race, body mass index, and other factors, an independent risk of diastolic blood pressure was found. Hypertensive women were $24 \%$ more likely to report fibroids, and the risk increased with duration of hypertension. The risk for fibroids also increased with the degree of hypertension. For every $10 \mathrm{~mm} \mathrm{HG}$ increase in diastolic blood pressure, the risk for fibroids increased $8 \%$ to $10 \%$. The increased pressure may be affecting uterine smooth muscle, causing damage with a similar mechanism as in vascular smooth muscle and hypertension.

\section{Infection and fibroids}

If fibroids may be triggered by myometrial injury as suggested [27], either through ischemia, pressure, or irritation from atherogenic-type mechanisms, such as hypertension, it is logical to inquire about the affect of infection on fibroid development. A case-control study of 318 women [28] that adjusted for hypertension, diabetes, age, ethnicity, body mass index, smoking, and oral contraceptive use, found a positive association with pelvic infection. A history of pelvic inflammatory disease increased the risk of fibroids, with the risk increasing with the number of infectious episodes. A history of three episodes of pelvic inflammatory disease conferred a RR of 3.7. Likewise, a history of Chlamydia conferred a RR of 3.2. Sexually transmitted diseases that mainly affected the external genitalia (genital warts and herpes) showed no association. It seems that the intrauterine irritation may contribute to the appearance or growth of fibroids.

Chagas' disease, the result of a parasitic infection endemic to portions of South America, has been reported to lead to an increased risk of various cancers. In an intriguing study [29] it was shown that the incidence of a positive history of Chagas' disease, diagnosed by serology, was significantly higher in women presenting for leiomyoma surgery. Twentyseven percent of women undergoing fibroid surgery had a serologically documented Chagas' infection versus $16 \%$ of controls. When the groups were further analyzed by race, white women with fibroids had a $40 \%$ prevalence of Chagas' versus $10 \%$ for nonwhite controls. It is known that Chagas' can parasitize the uterine smooth muscle, and this irritation may explain the association observed. 


\section{Smoking, alcohol, and caffeine}

It has been suggested that cigarette smoking could lower the risk of fibroids because it is associated with lower estrogen levels in some studies. The data are conflicting, with a RR of 1.6 for greater than one pack per day [2] to a decrease in risk (RR 0.7) [19,24]. In the well done Black Women's Health Study following almost 22,000 women there was no change in risk associated with tobacco smoking [30].

The Black Women's Health Study was able to demonstrate no change in risk related to caffeine consumption, but did see a small (RR 1.57) increase in risk for more than seven drinks of beer per week. Lesser amounts of beer and other alcohols showed smaller risks but did not reach statistical significance.

\section{Diet and fibroids}

One study [31] has specifically addressed the question of dietary influences on the prevalence of fibroids. In an Italian population, 843 women with fibroids were compared with 1557 women without. A diet weighted toward green vegetables was protective (RR 0.5 ), whereas a higher intake of meats was associated with a greater incidence of fibroids (RR 1.7). Given that diet is an essential component of lifestyle there are multiple confounding factors. This study does suggest, however, that lifestyle and environmental exposures seem to affect the incidence of fibroid disease.

\section{Summary}

Fibroids are a prevalent disorder occurring in at least half of American reproductive-age women. In general, the incidence and size increases with age. Most women never attribute or report any symptoms from their fibroids, and because of this the actual contribution of disease to symptoms of pelvic pain, menstrual symptoms, and infertility is poorly understood. The presence of fibroids can lead to multiple and disabling difficulties. Fibroids may cause pain and menstrual bleeding to the point of anemia. Fibroids clearly reduce fertility, increase preterm labor and delivery, and markedly increase the risk for cesarean delivery. Because the incidence varies according to population of interest, fibroids may explain some health disparities in different populations. For example, African Americans have a relatively poor outcome with assisted reproductive techniques compared with whites [32]. Controlling for fibroid disease may explain this disparity, at least in part [33].

Fibroids represent a tremendous public health burden on women and economic cost on society. Strategies to prevent, limit growth, and treat nonsurgically are needed. Fundamental and significant questions remain about fibroid disease, such as whether different clinical disease phenotypes (multiple versus single leiomyomas) contribute equally to symptoms and possess an equal likelihood of disease progression. For epidemiologic assessment of disease, a scoring system is urgently needed. Well-designed, controlled, prospective studies are still needed to define the natural history and correlate the presence of disease with symptomatology in women [34]. 


\section{Acknowledgments}

The authors acknowledge the helpful discussions of colleagues Dr. William Catherino, Dr. Lynnette Nieman, and Dr. John Tsibris. Support from Drs. Chrousos, Haffner, and Satin helped to make this work possible.

This research was supported, in part, by the Intramural Research Program of the Reproductive Biology and Medicine Branch, NICHD, National Institutes of Health.

\section{References}

1. Myers E, Barber M, Gustilo-Ashby T, et al. Management of uterine leiomyomata: what do we really know? Obstet Gynecol. 2002; 100:8-17. [PubMed: 12100798]

2. Chen C, Buck G, Courey N, et al. Risk factors for uterine fibroids among women undergoing tubal sterilization. Am J Epidemiol. 2001; 153:20-6. [PubMed: 11159141]

3. Baird D, Dunson D, Hill M, et al. High cumulative incidence of uterine leiomyoma in black and white women: ultrasound evidence. Am J Obstet Gynecol. 2003; 188:100-7. [PubMed: 12548202]

4. Aboyeji A, Ijaiya M. Uterine fibroids: a ten-year clinical review in Ilorin Nigeria. Niger J Med. 2002; 11:16-9. [PubMed: 12073294]

5. Heinemann K, Thiel C, Mohner S, et al. Benign gynecologic tumors: estimated incidence results of the German Cohort Study on Women's Health. Eur J Obstet Gynecol Reprod Biol. 2003; 107:7880. [PubMed: 12593900]

6. Marino J, Eskenazi B, Warner M, et al. Uterine leiomyoma and menstrual cycle characteristics in population based cohort study. Hum Reprod. 2004; 19:2350-5. [PubMed: 15242998]

7. Borgfeldt C, Andolf E. Transvaginal ultrasonographic findings in the uterus and endometrium: low prevalence of leiomyoma in a random sample of women age 25-40 years. Acta Obstet Gynecol Scand. 2000; 79:202-7. [PubMed: 10716301]

8. Sato F, Mori M, Nishi M, et al. Familial aggregation of uterine myomas in Japanese women. J Epidemiol. 2002; 12:249-53. [PubMed: 12164328]

9. Marshall L, Spiegelman D, Barbieri R, et al. Variation in the incidence of uterine leiomyoma among premenopausal women by age and race. Obstet Gynecol. 1997; 90:967-73. [PubMed: 9397113]

10. Wise L, Palmer J, Stewart E, et al. Age-specific incidence rates for self-reported uterine leiomyomata in the Black Women's Health Study. Obstet Gynecol. 2005; 105:563-8. [PubMed: 15738025]

11. Faerstein E, Szklo M, Rosenshein N. Risk factors for uterine leiomyoma: a practice based casecontrol study. I. African-American heritage reproductive history, body size, and smoking. Am J Epidemiol. 2001; 153:1-10. [PubMed: 11159139]

12. Farquhar C, Steiner C. Hysterectomy rates in the United States 1990-1997. Obstet Gynecol. 2002; 99:229-34. [PubMed: 11814502]

13. Sudik R, Husch K, Stellar J, et al. Fertility and pregnancy outcome after myomectomy in sterility patients. Eur J Obstet Gynecol. 1996; 65:209-14.

14. Launonen V, Vierimaa O, Kiuru M, et al. Inherited susceptibility to uterine leiomyomas and renal cell cancer. Proc Natl Acad Sci U S A. 2001; 98:3387-92. [PubMed: 11248088]

15. Muret G, Pujol R, Alomar A, et al. Familial leiomyomatosis cutis et uteri (Reed's syndrome). Arch Dermatol Res. 1998; 280:S29-32.

16. Pujol J, Pares D, Mora L, et al. Diagnosis and management of diffuse leiomyomatosis of the oesophagus. Dis Esophagus. 2000; 13:169-71. [PubMed: 14601911]

17. Toro J, Nickerson M, Wei M, et al. Mutations in the fumarate hydratase gene cause hereditary leiomyomatosis and renal cell cancer in families in North America. Am J Hum Genet. 2003; 73:95-106. [PubMed: 12772087]

18. Payson M, Segars J, Catherino W. Level of activating transcription factor 3 correlates with racial prevalence of leiomyoma. Fertil Steril. 2005; 84:S409. abstract.

19. Ross R, Pike M, Vessey M, et al. Risk factors for uterine fibroids: reduced risk associated with oral contraceptives. BMJ. 1986; 293:359-61. [PubMed: 3730804] 
20. DeWaay D, Syrop C, Nygaard I, et al. Natural history of uterine polyps and leiomyomata. Obstet Gynecol. 2002; 100:3-7. [PubMed: 12100797]

21. Hanafi M. Predictors of leiomyoma recurrence after myomectomy. Obstet Gynecol. 2005; 105:877-81. [PubMed: 15802421]

22. Baird, D. The NIEHS Uterine Fibroid Study: preliminary results. Presented at Advances in Uterine Leiomyoma Research: 2nd International Congress; Bethesda, Maryland. February 24-25, 2005;

23. Marshall L, Spiegelman D, Goldman M, et al. A prospective study of reproductive factors and oral contraceptive use in relation to the risk of uterine leiomyomata. Fertil Steril. 1998; 70:432-9. [PubMed: 9757871]

24. Parazzini F, Chiaffarino F, Polverino G, et al. Uterine fibroids risk and history of selected medical conditions liked with female hormones. Eur J Epidemiol. 2004; 19:249-53. [PubMed: 15117118]

25. Cesen-Cummings K, Copland J, Barrett J, et al. Pregnancy, parturition, and prostaglandins: defining uterine leiomyomas. Environ Health Perspect. 2000; 108(Suppl 5):817-20. [PubMed: $11035988]$

26. Boynton-Jarrett R, Rich-Edwards J, Malspeis S, et al. A prospective study of hypertension and risk of uterine leiomyomata. Am J Epidemiol. 2005; 161:628-38. [PubMed: 15781952]

27. Faerstein E, Szklo M, Rosenshein N. Risk factors for uterine leiomyoma: a practice based casecontrol study. II. Atherogenic risk factors and potential sources of uterine irritation. Am J Epidemiol. 2001; 153:11-9. [PubMed: 11159140]

28. Stewart E, Nowak R. New concepts in the treatment of uterine leiomyomas. Obstet Gynecol. 1998; 92:624-7. [PubMed: 9764641]

29. Murta E, Oliveira G, Prado F, et al. Association of uterine leiomyoma and Chagas' disease. Am J Trop Med Hyg. 2002; 66:321-4. [PubMed: 12139229]

30. Wise L, Palmer J, Harlow B, et al. Risk of uterine leiomyomata in relation to tobacco, alcohol, and caffeine consumption in the Black Women's Health Study. Hum Reprod. 2004; 19:1746-54. [PubMed: 15218005]

31. Chiaffarino F, Parazzini F, Vecchia C, et al. Diet and uterine myomas. Obstet Gynecol. 1999; 94:395-8. [PubMed: 10472866]

32. Grainger D, Seifer D, Frazier L, et al. Racial disparity in clinical outcomes from women using advanced reproductive technologies (ART): analysis of 80,196 ART cycles from the SART database 1999 and 2000. Fertil Steril. 2004; 82:S37-8.

33. Feinberg EC, Larsen FW, Catherino WH, et al. Comparison of ART utilization and outcomes between Caucasian and African American patients in an equal access to care setting. Fertil Steril. 2006 In Press.

34. Walker C, Stewart E. Uterine fibroids: the elephant in the room. Science. 2005; 308:1589-92. [PubMed: 15947177] 